\title{
Evaluación de la ingesta de líquido, pérdida de peso y tasa de sudoración en jóvenes triatletas

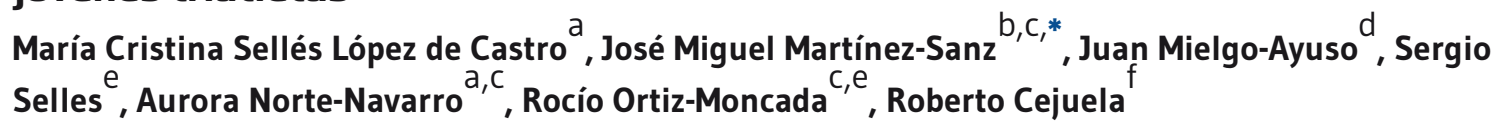

a Facultad de Ciencias de la Salud, Universidad de Alicante, España.

b Departamento de Enfermería, Facultad de Ciencias de la Salud, Universidad de Alicante, España.

C Gabinete de Alimentación y Nutrición, Universidad de Alicante (ALINUA), España.

d Imfine Research Group, Departamento de salud y rendimiento físico, Facultad de Ciencias de la Actividad Física y del DeporteInef, Universidad politécnica de Madrid, Madrid, España.

e Departamento de Enfermería Comunitaria, Medicina Preventiva y Salud Púbica e Historia de la Ciencia, Facultad de Ciencias de la Salud, Universidad de Alicante, España.

f Departamento de Didáctica General y Didácticas Específicas, Facultad de Educación, Universidad de Alicante, España.

*josemiguel.ms@ua.es

Recibido el 31 de diciembre de 2014; aceptado el 3 de junio de 2015.

Evaluación de la ingesta de líquido, pérdida de peso y tasa de sudoración en jóvenes triatletas

\author{
Deshidratación; \\ Deportes; \\ Triatlón; \\ Cambio de peso \\ corporal; \\ Sudor.
}

PALABRAS CLAVE

\section{RESUMEN}

Introducción: El triatlón es un deporte de resistencia que comprende tres disciplinas: natación, ciclismo y carrera a pie. Es necesario establecer pautas de hidratación para prevenir deshidrataciones durante entrenamientos o competiciones y mantener un buen estado de hidratación antes, durante y después del ejercicio. El objetivo de este estudio es evaluar la ingesta de líquido, pérdida de peso y tasa de sudoración en jóvenes triatletas, durante diferentes entrenamientos.

Material y Métodos: Estudio descriptivo-observacional en 14 triatletas (7 chicos y 7 chicas) durante una sesión de natación, otra de ciclismo y otra de carrera a pie. Se valoró la ingesta de líquido, pérdida de peso, \% agua corporal total, \% deshidratación y tasa de sudoración. Los triatletas bebieron agua en sus respectivos bidones de $750 \mathrm{ml}$ y se realizó una medición de orina en containers.

Resultados: Los resultados del estudio siguiendo el orden de natación, ciclismo y carrera a pie fueron: ingesta agua $2,66 \pm 1,94 \mathrm{ml} / \mathrm{min}, 7,91 \pm 7,69 \mathrm{ml} / \mathrm{min}$ y $7,08 \pm 4,13 \mathrm{ml} / \mathrm{min}$ en chi$\cos$ y $3,43 \pm 1,53 \mathrm{ml} / \mathrm{min}, 6,39 \pm 5,36 \mathrm{ml} / \mathrm{min}$ y $8,33 \pm 2,74 \mathrm{ml} / \mathrm{min}$ en chicas; pérdida de peso $0,83 \pm 0,5 \mathrm{~kg}, 0,47 \pm 0,3 \mathrm{~kg}$ y $0,98 \pm 0,4 \mathrm{~kg}$ en chicos y $0,79 \pm 0,3 \mathrm{~kg}, 0,47 \pm 0,58 \mathrm{~kg}$ y $0,28 \pm 0,21 \mathrm{~kg}$ en chicas; y tasa sudoración $4,44 \pm 4,9 \mathrm{ml} / \mathrm{min}, 11,81 \pm 6,46 \mathrm{ml} / \mathrm{min}$ y $5,29 \pm 3,13 \mathrm{ml} / \mathrm{min}$ en chicos y $3,89 \pm 2,4 \mathrm{ml} / \mathrm{min}, 4,69 \pm 4,20 \mathrm{ml} / \mathrm{min}$ y $7,96 \pm 5,06 \mathrm{ml} / \mathrm{min}$ en chicas.

Conclusiones: Se comparó el porcentaje de agua corporal y deshidratación, la pérdida de peso y la tasa de sudoración con otros estudios y se observa que nuestros resultados son inferiores a los estudios comparados, además están por debajo de la media de recomendaciones de líquido establecidas por el consenso de hidratación. 
$\sum$ Evaluation of fluid intake, weight loss and sweat rate in young triathletes

\section{KEYWORDS}

Dehydration;

Sports;

Triathlon;

Body weight

changes;

Sweat.

\section{CITA}

Sellés López de Castro MC, Martínez-Sanz JM, Mielgo-Ayuso J, Selles S, Norte-Navarro A, Ortiz-Moncada R, Cejuela R. Evaluación de la ingesta de líquido, pérdida de peso y tasa de sudoración en jóvenes triatletas. Rev Esp Nutr Hum Diet. 2015; 19(3): 132 - 139. DOI: 10.14306/renhyd.19.3.146.

\section{INTRODUCCIÓN}

El triatlón es un deporte olímpico que se practica sobre distintas distancias y comprende tres disciplinas de resistencia realizadas de forma consecutiva, siendo la más popular y ampliamente reconocida la que combina natación a estilo libre, ciclismo y carrera a pie ${ }^{1}$.

En la práctica del triatlón se deben controlar aspectos como la intensidad en cada segmento, la distribución del esfuerzo, la ingesta de nutrientes adecuada, y sobre todo, el control de una buena hidratación ${ }^{2}$. Para minimizar o evitar los efectos de la deshidratación, los atletas se deben asegurar de estar bien hidratados antes, durante y después del ejercicio ${ }^{3}$. Así, con el fin de prevenir posibles casos de deshidratación durante las pruebas que no beneficiarán a los deportistas, es necesario establecer unas pautas de hidratación $n^{4,5}$.
A pesar de que la práctica de triatlón va en aumento, existen pocas referencias que muestren los cambios en la composición corporal y balance hídrico durante la práctica de este deporte. Se ha mostrado cómo al finalizar un triatlón de larga distancia (Ironman) los deportistas pierden un 2,3\% de masa corporal ${ }^{6}$, mostrándose perdidas mayores en lugares con una mayor temperatura y humedad relativa.

Roger et al. concretaron, en un estudio realizado en un triatlón que consistió en $21 \mathrm{~km}$ de piragüismo, $97 \mathrm{~km}$ de ciclismo y $42 \mathrm{~km}$ de carrera a pie, que la pérdida de masa corporal fue de 1,9\% aproximadamente y correspondía a la tasa de sudoración (TS), producción de orina y pérdida de agua por la respiración?. Las ganancias de agua exógenas y endógenas sustituyeron cerca de un $90 \%$ de agua corporal total (ACT) perdida, por lo que es importante reponer los líquidos perdidos adecuadamente, ya que puede verse afectado tanto la salud como el rendimiento de los 
triatletas $^{7,8}$. La mayoría de los estudios que muestran estos cambios en la composición corporal se ha realizado, durante una competición como máxima expresión del rendimiento, sin mostrar los hábitos de hidratación de los deportistas durante el periodo de entrenamiento. Es de suma importancia conocer los hábitos de hidratación de los atletas para poder intervenir y concienciarlos de lo importante que es estar bien hidratado a la hora de realizar cualquier tipo de ejercicio físico ${ }^{9}$. La mejor opción es desarrollar un plan de ingesta de líquidos que se adapte a cada deporte y a las necesidades personales de cada deportista ${ }^{10}$.

Por ello, el objetivo de este estudio fue valorar la ingesta de líquido, pérdida de peso y TS en triatletas entrenados con un nivel de élite autonómico/nacional, durante diferentes entrenamientos de natación, ciclismo y carrera a pie.

\section{MATERIAL Y MÉTODOS}

\section{Participantes}

Se realizó un estudio descriptivo observacional sobre la ingesta de líquido, pérdida de peso y TS en 14 triatletas entrenados con un nivel de elite autonómico/nacional, 7 hombres $(20 \pm 2$ años, $175,5 \pm 3,9 \mathrm{~cm}$ y $70,5 \pm 4,2 \mathrm{~kg}$ ) y 7 mujeres $(22,43 \pm 3,15$ años, $162,1 \pm 6,9 \mathrm{~cm}$ y $58,2 \pm 7,1 \mathrm{~kg})$, respectivamente. Los triatletas estudiados entrenan un total de 15-25 horas/semana, repartidas en un total de 12-16 sesiones en función del período de la temporada en el que se encuentran. Los deportistas realizan doble o triple sesión de entrenamiento de lunes a viernes (natación en piscina, ciclismo, carrera a pie y fuerza). Los fines de semana realizan entrenamientos específicos de mayor volumen (natación aguas abiertas, ciclismo y carrera a pie). La carga de entrenamiento fue controlada por ECOs, según Cejuela y Esteve, 2011, un método específico de cuantificación de la carga de entrenamiento en triatlón, que relaciona el volumen, la intensidad y la densidad de los 3 deportes ${ }^{11}$. La carga media fue $823 \pm 112$ ECOs.

Todos fueron informados y dieron su consentimiento para participar en este estudio de acuerdo con la declaración de Helsinki 2013.

\section{Procedimiento e instrumentos}

La evaluación de los triatletas se llevó a cabo durante 3 sesiones de entrenamientos alternos a lo largo de una semana, 1 sesión de entrenamiento de natación de 120min de duración, 1 de ciclismo y otra de carrera a pie de 60min cada una. La sesión de natación tuvo lugar por la mañana de 13:00 a 15:00h en la piscina municipal de San Vicente del Raspeig (Alicante) con una temperatura ambiental de $31^{\circ} \mathrm{C}$ y humedad relativa de $63 \%$. La sesión de ciclismo y carrera a pie se realizó en horario de tarde de 16:00 a 17:00h en la Universidad de Alicante y alrededores, con una temperatura ambiental de $22^{\circ} \mathrm{C}$.

Para el registro del peso corporal, se siguió el protocolo elaborado por la International Society for the Advancement of Kinanthropometry ${ }^{12}$ (ISAK). Los triatletas fueron pesados en ropa interior y descalzos antes de iniciar el entrenamiento, habiéndoseles indicado que, en el caso de orinar o defecar, lo hicieran antes del pesaje inicial. Posteriormente al entrenamiento, los triatletas tuvieron que eliminar el sudor de sus caras, torso y piernas para volver a ser pesados. El \% de ACT se obtuvo antes del inicio del entrenamiento.

Para el registro del peso corporal y \% de agua ACT se utilizó la báscula-bioimpedancia Tanita BC-601 con fiabilidad del $97 \%$, precisión de 0,1 kg y con un rango de medida de 0-150kg. El \% de ACT se tomó antes del inicio de cada entrenamiento.

El cálculo del porcentaje de peso perdido se llevó a cabo mediante la siguiente fórmula tomada de Martins ${ }^{13}$ : Porcentaje (\%) Peso Perdido o Deshidratación $=[$ (Peso antes - Peso después) / Peso antes] x 100. Peso expresado en kg.

Para el cálculo del líquido total ingerido, cada triatleta contaba con un bidón de líquido con una capacidad de $750 \mathrm{ml}$ cada uno, llenos de agua.

Los triatletas fueron instruidos para que sólo bebieran de sus respectivos bidones, además de informarles que en el momento que necesitaran más líquido y el bidón estuviera vacío, se lo hicieran saber al investigador para Ilenarlo. Una vez finalizado el entrenamiento, el contenido de los bidones se vaciaba en una probeta. Al restar el volumen de líquido volcado en la probeta al volumen total de líquido que contenía el bidón, se obtiene la cantidad de líquido que ha consumido el triatleta durante el entrenamiento.

Para el registro del volumen de orina excretada por los triatletas, se midió el volumen de la misma desde el inicio de los diferentes entrenamientos hasta el final. Para ello los triatletas, antes del pesaje previo al entrenamiento, podían orinar sin necesidad de medir el volumen de orina excretada pero una vez realizado el primer pesaje, el triatleta debía orinar en un container estéril milimetrado y preparado para la recogida de orina con una capacidad de 2 litros.

El cálculo de la TS se llevó a cabo mediante la siguiente fórmula, tomada de Murray ${ }^{14}$ : Tasa Sudoración = (Peso Perdido + Líquido Ingerido - Orina) / Minutos Actividad. Peso expresado en $\mathrm{kg}$. 


\section{Análisis de datos}

Todos los datos son presentados como media y desviación estándar. Se comprobó la normalidad de todos los datos mediante la prueba de Shapiro-Wilk. Posteriormente, se realizó una ANOVA de dos factores (prueba y sexo) para conocer si había diferencias significativas entre ambos sexos y distintas pruebas en cada uno de los parámetros analizados. Las diferencias fueron consideradas estadísticamente significativas cuando $p<0,05$. Los análisis estadísticos se realizaron mediante el paquete estadístico SPSS ${ }^{\circledR}$, versión 22.0.

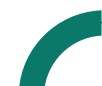

\section{RESULTADOS}

La Tabla 1 muestra que el líquido ingerido por parte de los triatletas en el entrenamiento de natación fue de $2,66 \pm 1,94 \mathrm{ml} / \mathrm{min}$, con una pérdida de peso de $0,47 \pm 0,32 \%$ (correspondiente a $0,67 \pm 0,46 \%$ ), una cantidad de agua corporal de $68,77 \pm 3,47 \%$ y una TS de $3,24 \pm 4,09 \mathrm{ml} / \mathrm{min}$. En esta misma tabla se muestran los datos derivados del entrenamiento de ciclismo de los triatletas, observándose

Tabla 1. Descripción de las variables relacionadas con la ingesta de líquido y grado de deshidratación en triatletas.

\begin{tabular}{|c|c|c|c|c|c|c|c|}
\hline & & Chicos $(n=7)$ & Rango & Sig. & Chicas $(n=7)$ & Rango & Sig. \\
\hline $\begin{array}{l}\text { Agua Corporal } \\
\text { (\%) }\end{array}$ & $\begin{array}{l}\text { Carrera a pie } \\
\text { Natación } \\
\text { Ciclismo } \\
\text { Total }\end{array}$ & $\begin{array}{l}65,75 \pm 0,92 \\
68,77 \pm 3,47 \\
65,70 \pm 1,32 \\
67,02 \pm 2,80\end{array}$ & $\begin{array}{l}65,10-66,40 \\
65,00-74,80 \\
64,80-68,30 \\
64,80-74,80\end{array}$ & 0,126 & $\begin{array}{l}63,38 \pm 1,93 \\
61,26 \pm 5,82 \\
65,10 \pm 3,94 \\
62,99 \pm 4,06\end{array}$ & $\begin{array}{l}59,90-65,30 \\
51,60-67,10 \\
62,30-69,60 \\
51,60-69,60\end{array}$ & 0,446 \\
\hline $\begin{array}{l}\text { Deshidratación } \\
\text { (\%) }\end{array}$ & $\begin{array}{l}\text { Carrera a pie } \\
\text { Natación } \\
\text { Ciclismo } \\
\text { Total }\end{array}$ & $\begin{array}{l}1,22 \pm 0,81 \\
0,67 \pm 0,46 \\
1,37 \pm 0,58 \\
1,05 \pm 0,62\end{array}$ & $\begin{array}{c}0,65-1,79 \\
0,07-1,30 \\
0,42-2,10 \\
0,07-2,10\end{array}$ & 0,126 & $\begin{array}{l}1,41 \pm 0,48 \\
0,65 \pm 0,79 \\
0,52 \pm 0,38 \\
0,95 \pm 0,69\end{array}$ & $\begin{array}{l}0,88-2,20 \\
-0,26-1,90 \\
0,11-0,85 \\
-0,26-2,20\end{array}$ & 0,080 \\
\hline $\begin{array}{l}\text { Pérdida peso } \\
(\mathrm{kg})\end{array}$ & $\begin{array}{l}\text { Carrera a pie } \\
\text { Natación } \\
\text { Ciclismo* } \\
\text { Total }\end{array}$ & $\begin{array}{l}0,83 \pm 0,53 \\
0,47 \pm 0,32 \\
0,98 \pm 0,43 \\
0,74 \pm 0,44\end{array}$ & $\begin{array}{l}0,45-1,20 \\
0,05-0,87 \\
0,30-1,45 \\
0,05-1,45\end{array}$ & 0,126 & $\begin{array}{l}0,79 \pm 0,30 \\
0,47 \pm 0,58 \\
0,28 \pm 0,21 \\
0,57 \pm 0,43\end{array}$ & $\begin{array}{c}0,50-1,15 \\
-0,15-1,40 \\
0,05-0,45 \\
-0,15-1,40\end{array}$ & 0,228 \\
\hline $\begin{array}{l}\text { Tasa Sudoración } \\
\text { (ml/min) }\end{array}$ & $\begin{array}{l}\text { Carrera a pie } \\
\text { Natación } \\
\text { Ciclismo } \\
\text { Total }\end{array}$ & $\begin{array}{l}5,29 \pm 3,13 \\
3,24 \pm 4,09 \\
11,81 \pm 6,46^{a} \\
7,20 \pm 6,39\end{array}$ & $\begin{array}{l}3,08-7,50 \\
-4,25-7,08 \\
5,83-22,50 \\
-4,25-22,50\end{array}$ & 0,045 & $\begin{array}{l}7,96 \pm 5,06 \\
5,45 \pm 4,05 \\
4,69 \pm 4,20 \\
6,36 \pm 4,44\end{array}$ & $\begin{array}{l}2,50-14,40 \\
0,83-11,66 \\
0,83-9,16 \\
0,83-14,40\end{array}$ & 0,530 \\
\hline $\begin{array}{l}\text { Líquido ingerido } \\
\text { (ml/min) }\end{array}$ & $\begin{array}{l}\text { Carrera a pie } \\
\text { Natación } \\
\text { Ciclismo } \\
\text { Total }\end{array}$ & $\begin{array}{l}7,08 \pm 4,13 \\
2,66 \pm 1,94 \\
7,91 \pm 7,69 \\
5,54 \pm 5,68\end{array}$ & $\begin{array}{l}4,16-10,00 \\
0,41-6,25 \\
0,00-20,83 \\
0,00-20,83\end{array}$ & 0,273 & $\begin{array}{l}8,33 \pm 2,74 \\
3,43 \pm 1,53 \\
6,39 \pm 5,36 \\
6,16 \pm 3,62\end{array}$ & $\begin{array}{l}5,00-12,50 \\
2,08-5,83 \\
2,50-12,50 \\
2,08-12,50\end{array}$ & 0,068 \\
\hline
\end{tabular}

Datos expresados en media \pm desviación estándar.

* Diferencias significativas entre sexos $(p<0,05)$.

Diferencias significativas entre pruebas: ( $\left.{ }^{a}\right)$ vs. carrera. 
una ingesta de líquido de $7,91 \pm 7,6 \mathrm{ml} / \mathrm{min}$, una pérdida de peso de $0,98 \pm 0,43 \mathrm{~kg}$ (correspondiente a $1,37 \pm 0,58 \%$ ), una ACT de $65,7 \pm 1,32 \%$ y una TS de $11,81 \pm 6,46 \mathrm{ml} / \mathrm{min}$. Por último, la Tabla 1 muestra que durante el entrenamiento de carrera a pie el líquido ingerido por parte de los triatletas fue de $7,08 \pm 4,1 \mathrm{ml} / \mathrm{min}$, con un peso perdido de $0,83 \pm 0,53 \mathrm{~kg}$ (correspondiente a $1,22 \pm 0,81 \%$ ), siendo la cantidad de agua total de $65,75 \pm 0,92 \%$ y la TS de $5,29 \pm 3,13 \mathrm{ml} / \mathrm{min}$.

También se observa que la ingesta de líquido en las triatletas durante el entrenamiento de natación fue de $3,43 \pm 1,53 \mathrm{ml} / \mathrm{min}$, con un $\%$ de deshidratación de $0,65 \pm 0,79 \%$ (correspondiente a $0,47 \pm 0,58 \mathrm{~kg}$ ), un $\%$ medio de agua corporal de $61,26 \pm 5,82$ y una TS de $5,45 \pm 4,05 \mathrm{ml} / \mathrm{min}$.

En el segundo entrenamiento, de ciclismo, la media de líquido ingerido fue de $6,39 \pm 5,36 \mathrm{ml} / \mathrm{min}$, con un $\%$ de deshidratación de 0,52 $\pm 0,38 \%$ (correspondiente a $0,28 \pm 0,21 \mathrm{~kg}$ de pérdida de peso), un $\%$ medio de agua corporal de 65,1 $\pm 3,94$ y una TS de 4,69 $\pm 4,20 \mathrm{ml} / \mathrm{min}$.

En el entrenamiento de carrera a pie, la media de líquido ingerido fue de $8,33 \pm 2,74 \mathrm{ml} / \mathrm{min}$, con un $\%$ de deshidratación de $1,41 \pm 0,48 \%$ (correspondiente a $0,79 \pm 0,3 \mathrm{~kg}$ de pérdida de peso) y una TS de $7,96 \pm 5,06 \mathrm{ml} / \mathrm{min}$.

La Tabla 2 muestra los resultados del peso antes del entrenamiento, peso después del entrenamiento y el peso después del entrenamiento menos el líquido ingerido en chicos y chicas para cada entrenamiento. Se puede observar tanto en chicos como en chicas que los pesos antes del entrenamiento en cada uno de ellos es mayor que los pesos después del entrenamiento, y éste, a su vez, es mayor que el peso después del entrenamiento donde se le ha restado el líquido ingerido. Esta pérdida de peso indica que ha habido pérdida de agua en los diferentes entrenamientos, aunque las diferencias no sean muy significativas. Se ha observado que en chicas, durante el entrenamiento de ciclismo el peso antes del entrenamiento es ligeramente inferior al peso de después del entrenamiento, pero este dato tampoco es muy significativo.

\section{Discusión y conclusiones}

Este estudio mostró el grado de deshidratación y la ingesta de líquidos por parte de un grupo de 14 triatletas de ambos sexos durante el entrenamiento de las distintas disciplinas que componen la práctica del triatlón. Los triatletas tuvieron un \% medio de deshidratación de $1 \%$ aproximadamente en ambos sexos y una ingesta media de líquidos para cada entrenamiento de $5,54 \pm 5,68 \mathrm{ml} / \mathrm{min}$ en chicos y $6,16 \pm 3,62 \mathrm{ml} / \mathrm{min}$ en chicas. Se destaca el \% de deshidratación en los triatletas durante la sesión de ciclismo $(1,37 \%)$ y en las triatletas durante el entrenamiento de carrera a pie $(1,41 \%)$. Se espera que la pérdida de peso por deshidratación no sobrepase el $2 \%$ para no comprometer el rendimiento deportivo ${ }^{15-17}$, tanto los hombres como las mujeres cumplieron con estas recomendaciones.

Tabla 2. Peso antes y después en los diferentes entrenamientos de los triatletas.

\begin{tabular}{|c|c|c|c|c|c|}
\hline & & \multicolumn{2}{|c|}{ Chicos $(n=7)$} & \multicolumn{2}{|c|}{ Chicas $(n=7)$} \\
\hline & & Media & Rango & Media & Rango \\
\hline $\begin{array}{l}\text { Peso antes del } \\
\text { entrenamiento }(\mathrm{kg})\end{array}$ & $\begin{array}{l}\text { Carrera a pie } \\
\text { Natación } \\
\text { Ciclismo }\end{array}$ & $\begin{array}{l}67,95 \pm 1,2 \\
70,53 \pm 4,23 \\
70,77 \pm 4,5\end{array}$ & $\begin{array}{l}67,1-68,8 \\
65-77,10 \\
64,8-77\end{array}$ & $\begin{array}{l}55,23 \pm 7,41 \\
65,5 \pm 9,65 \\
52,8 \pm 7,35\end{array}$ & $\begin{array}{l}45,6-66,3 \\
56,6-78,3 \\
45,3-60\end{array}$ \\
\hline $\begin{array}{l}\text { Peso después del } \\
\text { entrenamiento }(\mathrm{kg})\end{array}$ & $\begin{array}{l}\text { Carrera a pie } \\
\text { Natación } \\
\text { Ciclismo }\end{array}$ & $\begin{array}{l}67,55 \pm 1,48 \\
70,3 \pm 4,09 \\
70,27 \pm 4,49\end{array}$ & $\begin{array}{l}66,5-68,6 \\
64,4-76,7 \\
64,1-76,5\end{array}$ & $\begin{array}{l}54,95 \pm 7,22 \\
65,15 \pm 9,32 \\
52,9 \pm 7,5\end{array}$ & $\begin{array}{l}45,6-65,7 \\
57,2-78 \\
45,4-60,4\end{array}$ \\
\hline $\begin{array}{l}\text { Peso después del } \\
\text { entrenamiento menos } \\
\text { líquido ingerido }(\mathrm{kg})\end{array}$ & $\begin{array}{l}\text { Carrera a pie } \\
\text { Natación } \\
\text { Ciclismo }\end{array}$ & $\begin{array}{l}67,13 \pm 6,78 \\
69,93 \pm 3,76 \\
69,79 \pm 4,04\end{array}$ & $\begin{array}{l}65,9-68,35 \\
64,13-76,35 \\
64,1-75,95\end{array}$ & $\begin{array}{l}54,45 \pm 6,62 \\
64,81 \pm 8,15 \\
52,52 \pm 5,88\end{array}$ & $\begin{array}{l}45-65,2 \\
56,75-77,75 \\
49,25-59,65\end{array}$ \\
\hline
\end{tabular}


En hombres, durante el entrenamiento de ciclismo hay mayor TS (\%) y pérdida de peso $(\mathrm{kg})$ que en los entrenamientos de natación y carrera a pie. Ello podría ser debido a la mayor intensidad del entrenamiento e ingesta de líquidos en comparación con la de los otros entrenamientos.

En mujeres, durante el entrenamiento de carrera a pie hay mayor TS (\%) y pérdida de peso $(\mathrm{kg})$ que en los entrenamientos de natación y ciclismo, que podría ser debido a que tanto la ingesta de líquidos como la intensidad superan a los otros entrenamientos.

En un triatleta entrenado se espera que la pérdida de masa corporal en la realización de las competiciones, no supere el $3 \%{ }^{17}$. No obstante, la ingesta de líquido fue menor de lo recomendado tanto para el deporte en general como para el triatlón en particular. En este sentido, una protocolización adecuada de ingesta de líquidos será imprescindible para evitar una reducción del rendimiento deportivo ${ }^{18}$.

Durante el entrenamiento de ciclismo se puede observar que la pérdida de peso en chicos es mucho mayor que en chicas, y esto puede ser debido a una mejor adaptación y mayor tiempo de entrenamiento; a su vez, la TS es mayor en chicos que en chicas. Durante un Ironman, Mueller et al. ${ }^{19}$ mostraron una pérdida de peso media de $-1,9 \pm 0,8 \mathrm{~kg}$ en 8 triatletas varones. Sin embargo, en nuestro estudio mostramos una pérdida de peso inferior en todos los entrenamientos, siendo el resultado más alto en hombres para el entrenamiento de ciclismo $(0,98 \pm 0,43 \mathrm{~kg})$. Por otro lado, Speedy et al. ${ }^{20}$ durante el Ironman de Nueva Zelanda de 1997, que correspondía a $3,8 \mathrm{~km}$ de natación, $180 \mathrm{~km}$ de ciclismo y $42,2 \mathrm{~km}$ de carrera a pie, estudiaron a 12 hombres y 7 mujeres cuya edad y peso medio fueron de 35 años y $67 \mathrm{~kg}$ de peso. Durante la competición la pérdida de peso fue de $-1 \mathrm{~kg}$ durante la prueba de natación, $-2 \mathrm{~kg}$ durante la de carrera a pie, mientras que se observó un aumento de $0,5 \mathrm{~kg}$ de peso en ciclismo. En eventos de triatlón de larga distancia se ha observado cambios de peso corporal que varían entre - $10,7 \%$ y +3,7\% ${ }^{17}$, debidos a la sudoración y utilización de sustrato energético. Mientras tanto, en nuestro estudio, se observó en cada tipo de entrenamiento una pérdida de peso pero sin que esta disminución superará las mostradas por otros autores ${ }^{19,20}$.

Por otro lado, Pfeiffer et al. ${ }^{21}$ midieron la ingesta de líquidos en diferentes entrenamientos de pruebas de resistencia incluido el triatlón. Se observó que los atletas ingirieron durante distintas competiciones de ciclismo $\sim 1.200 \mathrm{ml} / \mathrm{h}^{-1}$ y $6.700 \pm 200 \mathrm{ml} / \mathrm{d}^{-1}$ cuyos valores corresponden al Tour de Francia durante 5 horas y 14 minutos, y $600 \pm 178 \mathrm{ml}^{-1} \mathrm{~h}^{-1}$ cuyo valor corresponde al evento Road Race durante 7 horas y 18 minutos. Durante la carrera a pie fue de $765 \mathrm{ml} / \mathrm{h}^{-1}$, $740 \mathrm{ml} / \mathrm{h}^{-1}$ y $540 \pm 210 \mathrm{ml} / \mathrm{h}^{-1}$ cuyos tres valores corresponden a una Ultramaratón de $160 \mathrm{~km}$ los dos primeros y $100 \mathrm{~km}$ el último, realizados en 24 horas y 18 minutos, 26 horas y 12 minutos y 10 horas y 29 minutos. Además, analizaron las ingestas medias de líquido en diferentes eventos de Ironman ${ }^{20}\left(354-794 \mathrm{ml} / \mathrm{h}^{-1}\right)$. En la misma línea, otros estudios llevados a cabo en distintos eventos de triatlón a lo largo del mundo en diferentes años ${ }^{22}$ mostraron ingestas de líquido entre $540 \mathrm{ml} / \mathrm{h}^{-1}$ y $700 \mathrm{ml} / \mathrm{h}^{-1}$. A pesar de que los porcentajes de deshidratación obtenidos por nuestros triatletas se sitúan dentro del margen de lo establecido, en ningún caso se alcanzó la cantidad recomendada de líquido, tanto a nivel general (400-500ml líquido/h) como especifico en triatlón (>600ml líquido/hora) ${ }^{5,16}$. Esto nos lleva a pensar en una recomendación sobre ingesta de líquidos, habituando al organismo a ingerir más cantidad de líquido durante el entrenamiento ${ }^{4}$, ya que la media de ingesta de líquidos tanto en hombres como en mujeres está por debajo de todas las medias de los diferentes artículos comparados.

Un deportista adulto debe de situarse por encima del 60\% de ACT por su elevada masa magra, baja proporción de grasa y elevado contenido en glucógeno muscular ${ }^{23}$. Datos superiores observamos en todos los deportistas de nuestro estudio, situándose por encima del 64-65\%, a excepción de las mujeres que mostraron valores similares al de la población general en el entrenamiento de natación realizado (59,8 $\pm 5,56 \%)$. Los diferentes resultados de ACT entre sexos pueden ser debidos a que los triatletas varones poseen de forma general mayor masa magra ${ }^{24}$ que las féminas. Otra de las posibles causas sería las estrategias de hidratación previas al entrenamiento o ingesta de líquidos durante todo el día.

Respecto a la TS, en un estudio realizado a jugadores de fútbol sala durante una competición oficial, la media de la TS del partido fue de $43,83 \pm 14,7 \mathrm{ml} / \mathrm{min}$. Sin embargo, en nuestro estudio se observaron TS inferiores tanto en los hombres (4,44-11,81 ml/min) como en las mujeres (3,89-7,96ml/min), en comparación con los jugadores de fútbol sala que a pesar de tener una TS y la ingesta de líquidos mayor, alcanzándose valores de deshidratación mínimos sólo se vio afectado el incremento cardiaco y algunas condiciones motoras ${ }^{25}$. Se puede observar en nuestro estudio que la TS durante el entrenamiento de ciclismo en chicos, es mucho mayor que durante los entrenamientos de carrera a pie y natación. Esto puede ser debido a una mayor intensidad y esfuerzo durante el entrenamiento y a que no fue cubierto con la hidratación necesaria por los triatletas, teniendo en cuenta que a mayor intensidad mayores pérdidas de glucógeno que pueden influir en la deshidratación.

Por ello es importante realizar la valoración del estado de hidratación e ingesta de líquidos durante los entrenamientos, 
con el fin de protocolizar y establecer las mejores estrategias hídricas según cada individuo. Además, en actividades deportivas con duración inferior o aproximada a 1 hora, la reposición de fluidos suele ser difícil, porque la intensidad del esfuerzo suele ser muy elevada (por encima del 75-80\% del $\mathrm{VO}_{2}$ máx) y el ritmo de reposición hídrica teórica necesaria para compensar las pérdidas puede producir molestias digestivas. También la toma de líquidos puede afectar en la biomecánica de la carrera, así como disminuir el ritmo en momentos para realizar la ingesta de líquidos ${ }^{4}$.

Algunas de las limitaciones del presente estudio son el número reducido de la muestra así como el número de sesiones de entrenamiento valoradas. También podemos citar como limitaciones la variación del peso en el entrenamiento de natación al pesar a los atletas en traje de baño, no se tuvo en cuenta la hidratación a lo largo del día y no se midió mediante el color de la orina intentando ser solucionado mediante la valoración de ACT por bioimpedancia eléctrica. Por otra parte, se ha descrito en la literatura científica la pérdida de peso, ingesta de líquidos y deshidratación en triatletas durante la competición, especialmente en larga distancia como el Ironman, pero no encontramos estudios desarrollados durante los entrenamientos, salvo los que se han encontrado en deportes de equipo.

Como conclusiones prácticas que aporta el presente estudio son: 1. Realizar programas de intervención en triatletas para elaborar estrategias de ingesta de líquidos basadas en las características individuales; 2 . La valoración de la ingesta de líquidos, pérdida de peso y TS es importante para conocer la ingesta hídrica que debe realizar el triatleta según el tipo de entrenamiento, para ayudar al balance hídrico y estado de hidratación en el entrenamiento ${ }^{26}$; y 3. Estas valoraciones deben formar parte de las estrategias dietéticonutricionales para el triatleta, que el dietista-nutricionista deportivo y cuerpo médico debe desarrollar como parte de sus competencias ${ }^{27}$.

\section{$\longrightarrow$ \\ AGRADECIMIENTOS}

Agradecer a los triatletas y entrenadores del equipo de Tecnificación de Triatlón de la Universidad de Alicante, por su consentimiento en participar en este estudio.

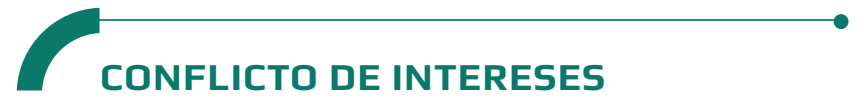

El presente artículo es un texto original y no presenta conflictos de intereses de tipo económico con instituciones, organizaciones u autores.

\section{BIBLIOGRAFÍA}

1. www.triatlón.org [portal de internet] Federación Española de Triatlón (FETRI). España: Reglamento de competiciones [actualizado 19 feb 2011; citado 22 Abril 2014]. Disponible en: http://triatlon.org/triweb/wpcontent/uploads/2013/09/ FETRI.2011.Reglamento-de-Competiciones.pdf

2. www.elsitiodelagua.com [portal de internet] El sitio del agua. Fundación Mondariz Balneario, España: Hidratación y electrolitos durante el Ironman citado [5 Mayo 2014]. Disponible en: http://www.elsitiodelagua.com/i/biblioteca/ CH_0005.pdf

3. Reinoso Ruiz JR. Alimentación pre-competitiva y postcompetitiva en el triatlón olímpico y de larga distancia. Journal of Teaching: didáctica del profesor. 2009; 1: 21-34.

4. Urdampilleta A, Martínez-Sanz JM, Julia Sanchez S, Alvarez Herms J. Protocolo de hidratación antes, durante y después de la actividad físico-deportiva. Mot Eur J Hum Mov. 2013; 31: 57-76.

5. Jeukendrup AE. Nutrition for endurance sports: marathon, triathlon, and road cycling. J Sports Sci. 2011; 29(Suppl 1): S91-9

6. Rogers G, Goodman C, Rosen C. Water budget during ultraendurance exercise. Med Sci Sports Exerc. 1997; 29(11): 1477 81.

7. Speedy DB, Noakes TD, Kimber NE, Rogers IR, Thompson JM, Boswell DR, et al. Fluid balance during and after an Ironman triathlon. Clin J Sport Med. 2001; 11(1): 44-50.

8. González-Alonso J, Coyle EF. Efectos fisiológicos de la deshidratación. ¿Por qué los deportistas deben ingerir líquidos durante el ejercicio en el calor? Ed Física y Deportes. 2009; 54: 46-52.

9. García Jiménez JV, Yuste Lucas JL, García Pellicer J]. Ingesta de líquidos y deshidratación en jugadores profesionales de fútbol sala en función de la posición ocupada en el terreno de juego. Apunts Med Esport. 2010; 45: 69-74.

10. Kent $M$, Schamasch P. Nutrición para deportistas. 2 $2^{a}$ ed. Lausana; 2012.

11. Cejuela Anta R, Esteve-Lanao J. Training load quantification in triathletes. J Hum Sport Exerc. 2011; 6(2): 218-32.

12. ISAK. International Standars for Anthropometric Assessment. Glasgow: International Society for Advancement of Kinanthropometry; 2011.

13. Martins M, Aparecida J, Kleverson J, Works RH, Wagner R, Bohn JH, et al. A desidrataçao corporal de atletas amadores de futsal. Revista Brasileira de Prescriçao e Fisiologia do Exercício. 2007; 1(5): 24-36. 
14. Murray R. Hydration and physical performance. J Am Coll Nutr. 2007; 26(5Suppl): 542S-8S.

15. Urdampilleta A, Gómez-Zorita S. De la deshidratación a la hiperhidratación: bebidas isotónicas y diuréticas y ayudas hiperhidratantes en el deporte. Nutr Hosp. 2014; 29: 21-5.

16. Jeukendrup $A E$, Jentjens RLPG, Moseley $L$. Nutritional considerations in triathlon. Sports Med. 2005; 35(2): 163-81.

17. Laursen PB. Long distance triathlon: demands, preparation and performance. J Hum Sport Exerc. 2011; 6(2): 247-63.

18. García-Jiménez JV, Yuste JL. Tasa de sudoración y niveles de deshidratación en jugadores profesionales de fútbol sala durante competición oficial. Arch Med Deporte. 2010; (27): 457-64.

19. Mueller SM, Anliker E, Knechtle P, Knechtle B, Toigo M. Changes in body composition in triathletes during an Ironman race. Eur J Appl Physiol. 2013; 113(9): 2343-52.

20. Speedy DB, Noakes TD, Kimber NE, Rogers IR, Thompson JM, Boswell DR, et al. Fluid balance during and after an Ironman triathlon. Clin J Sport Med. 2001; 11(1): 44-50.

21. Pfeiffer B, Stellingwerff $T$, Hodgson AB, Randell R, Pöttgen $K$, Res $P$, Jeukendrup AE. Nutritional intake and gastrointestinal problems during competitive endurance events. Med Sci Sports Exerc. 2012; 44(2): 344-51.

22. Getzin AR, Milner C, LaFace KM. Nutrition update for the ultraendurance athlete. Curr Sports Med Rep. 2011; 10(6): 330-9.

23. Iglesias Rosado C, Villarino Marin AL, Martinez JA, Cabrerizo $L$, Gargallo M, Lorenzo $H$, et al. Importancia del agua en la hidratación de la población española: documento FESNAD 2010. Nutr Hosp 2011; 26: 27-36.

24. Cabañas Armesilla MD, Esparza Ros F. Compendio de cineantropometria. Madrid: CTO; 2009.

25. Barberó JC, Castagna C, Granda J. Deshidratación y reposición hídrica en jugadores de fútbol sala: efectos de programa de intervención sobre la pérdida de líquido durante la competición. Mot Eur ] Hum Mov. 2006; 17: 97-110.

26. Evans GH, Shirreffs SM, Maughan RJ. Postexercise rehydration in man: the effects of osmolality and carbohydrate content of ingested drinks. Nutrition. 2009;25 (9): 905-13.

27. Belloto M, Palma-Linares I. Las competencias nutricionales del nutricionista deportivo. Rev Nutr. 2008; 21: 633-46. 